\title{
Dynamical computation reservoir emerging within a biological model network
}

\author{
Carlos Lourenço ${ }^{1}$ \\ Faculty of Sciences of the University of Lisbon - Informatics Department \\ Campo Grande, 1749-016 Lisboa - Portugal \\ and \\ Instituto de Telecomunicações - Security and Quantum Information Group \\ Av. Rovisco Pais, 1, 1049-001 Lisboa - Portugal
}

\begin{abstract}
Chaos in dynamical systems potentially provides many different dynamical states arising from a single attractor. We call this the reservoir property and give here a precise meaning to two aspects of such property. In both cases, the high flexibility of chaos comes into play, as compared to more regular regimes. In this article, we especially focus on the fact that chaotic attractors are known to possess an infinite number of embedded Unstable Periodic Orbits. In brain modeling, or for the purpose of suggesting computational devices that could take advantage of chaos, the different embedded dynamical states can be interpreted as different behaviors or computational modes suitable for particular tasks. Previously we proposed a rather abstract neural network model that mimicked cortex to some extent but where biological realism was not the major concern. In the present paper we show that the same potential for computation can be displayed by a more realistic neural model. The latter features spatiotemporal chaos of a type thus far only found in more "artificial" models. We also note that certain network-related properties, previously overlooked, turn out to be essential for the generation of complex behavior.
\end{abstract}

Key words: chaos, dynamical reservoir, computation, spatiotemporal dynamics, self-organization, neurodynamics

PACS: 05.45.Gg, 05.45.Pq, 05.45.Xt, 05.65.+b, 87.10.+e, 87.18.Bb, 87.18.Hf, 87.18.Sn, 87.19.La, 89.75.Fb

Email address: csl@di.fc.ul.pt (Carlos Lourenço).

URL: http://www.di.fc.ul.pt/ csl/ (Carlos Lourenço).

1 The author acknowledges the partial support of Fundação para a Ciência e a Tecnologia and EU FEDER via the former Center for Logic and Computation and the project ConTComp (POCTI/MAT/45978/2002), and also via the project $\mathrm{PDCT} / \mathrm{MAT} / 57976 / 2004$. 


\section{Spatiotemporal neural chaos and computation}

From the mid-1980s onward, different groups reported the discovery of chaotic electrical signals in the brain, namely human [1] and simian [2]. This started a long-lasting dispute concerning the true chaotic nature of such signals, as well as much speculation regarding the possible roles of chaos in cognition [3-6]. Our standpoint in previous work and in the present paper is as follows. We take chaos for a fact and assume that natural systems may display it, including in the biological case. We then ask what computational purposes it may serve, and which of those might be relevant for biologically inspired computational devices. In this vein, actual computational models were proposed [6-10]. These particular models adopt a continuous-time setting and feature nonlinear network properties. The investigation of the neurons' dynamics is assisted by general knowledge of properties of nonlinear oscillators, as well as of generic networks. In the references above, rather abstract models such as GinzburgLandau and Rossler oscillators are meant to capture the essential oscillatory features of neurons. Particularly in $[6,7,9]$, a full network setting is presented mimicking cortical architecture. Thus an actual spatiotemporal dynamics is unveiled, overcoming the limitations and criticism that result from working with single-unit or otherwise very small networks [9]. Unstable Periodic Orbits (UPOs) can be stabilized from within chaos, very fast and with minimum perturbation of the original system. The original chaotic attractor contains an infinite number of such dynamical modes, some of which can be stabilized at will according to the requirements of computational tasks. In [9], this is applied to the processing of spatiotemporal visual input patterns with different symmetries. The units (or "neurons") are topologically arranged in a network, and the simultaneous monitoring of their state variables reveals such spatiotemporal regimes as standing and rotating waves of different symmetries, or a complex mixture thereof. The name "reservoir" in the context of a chaotic computational device has been adopted in [6] and later references by the same authors, where it refers explicitly to the fact that chaos can contain an infinite number of UPOs and these can be used as coding devices or computational modes. Interestingly, the concrete application of chaotic computing featured in $[6,7,9]$ is also an early, albeit partial, demonstration of what is modernly called reservoir computing. The latter is featured namely in Echo State Networks [11] and Liquid State Machines [12], where the dynamics of a large pool of neurons in a recurrent network is perturbed by some input and the resulting transient dynamics is assessed by separate readout neurons as a process which can provide meaning to the observed dynamics. However, and apart from other differences, the readout mechanism in $[6,7,9]$ is at a more basic level and in particular it lacks the learning capabilities of $[11,12]$. The fact that in $[6,7,9]$ a chaotic attractor is perturbed, either in a permanent or a transient fashion, allows the use of a large range of resulting system responses. This also suggests the term "reservoir" as referring to the availability 
of a rich collection of dynamical input-output transformations, thus in closer agreement with the spirit of $[11,12]$. This somehow conflicts with the use of the word in the sense of a reservoir of UPOs, and is not exactly the meaning inherent to the so-called reservoir computing if in the latter case the reservoir is understood as the pool of neurons itself. Notwithstanding, this explanation should clarify the term usage in each context. In the two senses of reservoir of UPOs and reservoir of responses, the flexibility of chaos is stressed out. Note that the major theme of the present paper is the reservoir of UPOs itself. This constitutes an important module of a system demonstrating computation with perturbations under our model, but a complete application is out of the scope of the present publication. The reader interested in previewing what the final system will look like, should presently be referred to the device in [9]. Although it uses a more abstract model, it does display a number of features which will look familiar to those acquainted with Liquid Sate Machines and similar devices. Namely, a fading memory is present, and the system is perturbed differently according to specific characteristics of each input, for both cases of static and dynamic input.

\section{Nonlinear oscillators: from abstract units to more realistic neu- rons}

The mathematical models mentioned above face some criticism when comparisons are made with actual neurons. This is the case with the models in [6-10], but also, in varying degree, with other models in the literature. Neurons are not oscillators, even if certain cells can display autonomous rhythmic firing (a behavior we are not addressing here, but certainly nothing like an actual oscillator, especially if the membrane potential is the monitored variable). However, groups of neurons can show oscillating electrical activity, sustained by the exchange of excitation and inhibition. Neural coupling is far from the simple linear connectivity of diffusive type considered in $[6,7,9]$ and other studies of networks of the reaction-diffusion type. Rather, neurons are connected via highly nonlinear transfer functions such as sigmoids. Finally, in real life there is an unavoidable delay in signal transmission between all neurons, which is usually not considered as an intrinsic property of the model networks. This includes the networks mentioned above.

Hence a more realistic model is sought. Our purpose is to attain just the "right" level of biological or physical plausibility, while still having a manageable model for dynamical exploration. Although proposed in a different context, the model appearing in [13] and further developed in [14] provides a good compromise. The individual unit is a slightly more complex version of the leaky integrator, and is also called the single-compartment neuron. Passive as well as active membrane properties are considered, along with highly 
nonlinear coupling and delays in signal transmission between neurons. While aiming at biological realism, the model that we adopt also turns out to fit in the general framework of the Kleinfeld model [15], which has been proposed as a standard model for delayed recurrent neural networks [16]. The model is amenable to synaptic learning, namely by backpropagation [16], but we have not considered that possibility in our own work thus far. An abbreviated account of the UPO reservoir properties of this model was given in [17].

\subsection{Deriving the neuron model}

The detailed morphology of the neuron need not be taken into account as it would be the case with more complex models with multiple compartments. The emphasis of the modeling is on the overall electrical state of each neuron, and not on the spatial distribution of charge within a neuron. We start with the electrical equivalent of the neural membrane as illustrated by Fig. 1. The

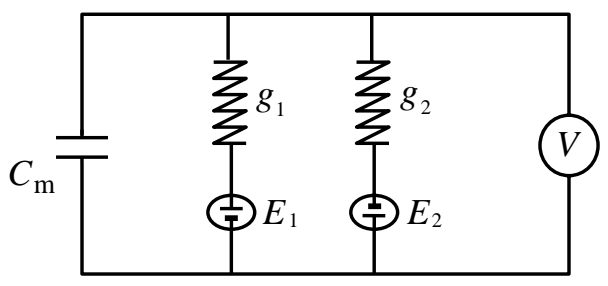

Fig. 1. Electrical equivalent of the neural membrane. See text for the details.

state of the neuron is characterized by the membrane potential $V . C_{\mathrm{m}}$ is the membrane capacitance. $E_{n}$ represents the ionic potential associated with ion species $n$. The membrane conductance associated with species $n$ is denoted $g_{n}$. A Resistive-Capacitive (RC) equation can be written for the potential $V$ :

$$
C_{\mathrm{m}} \frac{d V}{d t}=-g_{1}\left(V-E_{1}\right)-g_{2}\left(V-E_{2}\right)
$$

Actually $E_{1}$ and $E_{2}$ can be taken as linear combinations of different ionic potentials [13], but we need not detail them here as the setting is general enough. The ionic conductances are decomposed into a "passive" and an "activated" part,

$$
g_{n}=g_{n}^{0}+g_{n}^{c} .
$$

The resting conductance $g_{n}^{0}$, specific to species $n$, is constant in time. The activated part, $g_{n}^{c}$ for each species $n$, takes into account the opening or closing of ionic channels, depending on the level of synaptic excitation which arises through the coupling of neurons. We may write 


$$
\begin{aligned}
g_{L} & =g_{1}^{0}+g_{2}^{0} \\
V_{L} & =\frac{g_{1}^{0} E_{1}+g_{2}^{0} E_{2}}{g_{L}}
\end{aligned}
$$

where $g_{L}$ is the resting conductance of the membrane and $V_{L}$ is its resting potential. The previous $\mathrm{RC}$ equation can thus be transformed into

$$
C_{\mathrm{m}} \frac{d V}{d t}=-g_{L}\left(V-V_{L}\right)-g_{1}^{c}\left(V-E_{1}\right)-g_{2}^{c}\left(V-E_{2}\right)
$$

Let $g_{n}^{(k \rightarrow i)}$ denote the local conductance of ion species $n$, at the synapse between neurons $k$ and $i$. Then, we have

$$
g_{n, i}^{c}=\sum_{k} g_{n}^{(k \rightarrow i)}
$$

integrating the contributions from several presynaptic neurons, indexed by $k$. While $g_{n}^{(k \rightarrow i)}$ depends on the stimulation provided by neuron $k$, we neglect its dependence on the voltage $V$ of cell $i$ itself. The different equilibrium potentials have the values $V_{L}=-60 \mathrm{mV}, E_{1}=50 \mathrm{mV}$, and $E_{2}=-80 \mathrm{mV}$. As with other parameters of the model, these values are suggested by electrophysiological experiments. The activation of channel $g_{1}^{c}$ contributes to the increase of the membrane potential toward $E_{1}$, whereas the activation of $g_{2}^{c}$ tends to decrease the membrane potential toward $E_{2} . g_{1}^{c}$ and $g_{2}^{c}$ are activated by excitatory and inhibitory presynaptic neurons, respectively. The synaptic conductances depend on the firing rate of the presynaptic neuron:

$$
g_{n}^{(k \rightarrow i)}(t) \propto f_{k}\left(t-\tau_{i k}\right)
$$

where $\tau_{i k}$ is the time-delay due to the finite speed of signal propagation. The firing frequency of the presynaptic neuron $k$ saturates at a value $f_{\max }$, say, $100 \mathrm{~Hz}$. We consider a sigmoidal transfer function between the membrane potential $V_{k}$ and the firing frequency $f_{k}$ :

$$
\begin{aligned}
f_{k} & =f_{\max } F\left(V_{k}\right) \\
F(V) & =\frac{1}{1+e^{-\alpha\left(V-V_{c}\right)}} .
\end{aligned}
$$

The transfer function $f$ is illustrated in Fig. 2. By choosing particular parameter values $\alpha$ and $V_{c}$, a certain activation threshold is obtained such that the neuron is silent for lower values of the potential $V$. Past some higher value of $V$, the firing rate saturates. $V_{c}$ is fixed at $-25 \mathrm{mV}$. Two different $\alpha$ values are considered as indicated in Section 2.2. 


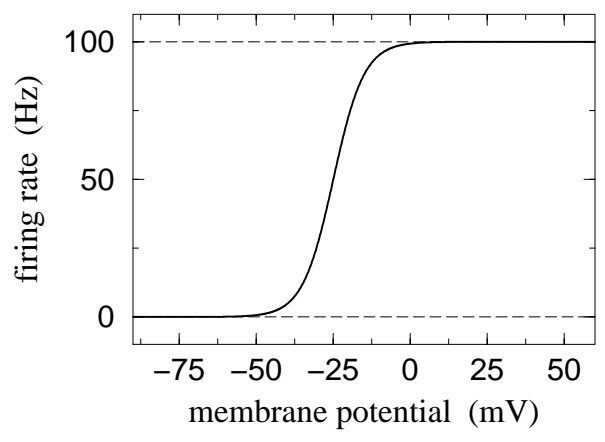

Fig. 2. Dependence of a neuron's instantaneous firing rate on its own membrane potential. Parameters of the sigmoid $f$ in this figure are $\alpha=0.2 \mathrm{mV}^{-1}, V_{c}=-25 \mathrm{mV}$, $f_{\max }=100 \mathrm{~Hz}$.

\subsection{Connecting the neurons}

In the isolated model neuron, the response of the membrane to perturbations from the resting state can be characterized by $\gamma=g_{L} / C_{\mathrm{m}}$, which is the inverse of the membrane's time-constant. The dynamics in this simple case is relaxatory. However, as several neurons are coupled together, new dynamical regimes may settle in, depending on details of the coupling and on the initial conditions of the population. One can observe multiple steady states, including global quiescence and global saturation, as well as a variety of oscillatory regimes for the electrical activity of the neurons. Although a single neuron, under the present model, does not oscillate, a coupled population may present oscillations due to the interplay of excitatory and inhibitory feedback through synaptic connections.

The variables of the dynamics are the instantaneous membrane potential of each neuron. A stationary state corresponds to the firing of action potentials with a frequency constant in time. This frequency can be close to zero, or have some finite value. An oscillatory state, on the other hand, implies a timemodulation of the firing frequency.

Although there are many types of neurons, for the purpose of the modeling we divide them into two populations: one of excitatory neurons, with membrane potentials $X_{i}$, and another of inhibitory neurons, with membrane potentials $Y_{j}$. The dynamics of the coupled neuronal populations is described by 


$$
\begin{aligned}
\frac{d X_{i}}{d t}= & -\gamma\left(X_{i}-V_{L}\right)-\left(X_{i}-E_{1}\right) \sum_{k \neq i} \omega_{i k}^{(1)} F_{X}\left[X_{k}\left(t-\tau_{i k}\right)\right] \\
& -\left(X_{i}-E_{2}\right) \sum_{l \neq i} \omega_{i l}^{(2)} F_{Y}\left[Y_{l}\left(t-\tau_{i l}\right)\right] \\
\frac{d Y_{j}}{d t}= & -\gamma\left(Y_{j}-V_{L}\right)-\left(Y_{j}-E_{1}\right) \sum_{k \neq j} \omega_{j k}^{(3)} F_{X}\left[X_{k}\left(t-\tau_{j k}\right)\right] \\
& i, k=1, \ldots, N_{\mathrm{ex}}, \quad j, l=1, \ldots, N_{\text {in }} .
\end{aligned}
$$

The network comprises $N_{\text {ex }}$ excitatory neurons $X_{i}$, and $N_{\text {in }}$ inhibitory neurons $Y_{j}$. The inverse of the membrane's time-constant takes the value $\gamma=$ $0.25 \mathrm{msec}^{-1}$. The propagation delay $\tau_{i k}$ between neurons $k$ and $i$ depends on the distance between the two neurons. Contrarily to previous versions of the model, here we consider different values of the slope $\alpha$ of the transfer function $F$, depending on whether the firing neuron is excitatory or inhibitory. We adopt the values $\alpha_{X}=0.09 \mathrm{mV}^{-1}$ and $\alpha_{Y}=0.2 \mathrm{mV}^{-1}$. The synaptic weights $\omega$ characterize the efficiency of channel activation by the presynaptic neuron. They correspond to the coupling constants of the network. The weights $\omega_{i k}^{(1)}, \omega_{i l}^{(2)}$, and $\omega_{j k}^{(3)}$ refer, respectively, to excitatory-to-excitatory, inhibitoryto-excitatory, and excitatory-to-inhibitory connections. We take these values as constant in time. No inhibitory-to-inhibitory connections are considered.

The connectivity in the model is of a local nature. For simplicity, only firstneighbor connections are implemented. Furthermore, we study networks that are invariant under translations in space, where the neurons are equally spaced. Therefore, in the $X_{i}$ and $Y_{j}$ equations one can drop the subscripts in the time-delays and synaptic weights: $\tau_{i k}=\tau$ and $\omega_{i k}^{(I)}=\omega^{(I)}$, where $I=1,2,3$ designates the different types of interactions. The summations in the $X_{i}$ and $Y_{j}$ equations are to be performed with contributions only from presynaptic neurons in the immediate spatial vicinity of the postsynaptic neuron. This local pattern of connectivity may be more relevant for certain parts of the cortex than for others, but this discussion is out of the scope of the present paper.

\section{$2.3 \quad$ Network topology}

We have investigated the dynamical behavior of the above equations in different configurations, including the case of bi-dimensional networks with many excitatory and inhibitory neurons. However, for the purpose of this paper, we consider only a 1-D spatial arrangement of a moderate number of neurons. Spatiotemporal chaotic solutions are sought, as well as embedded UPOs therein. These UPOs are expected to display diverse spatiotemporal symmetries and could eventually be stabilized via suitable control methods. Figure 3 
shows the network topology. Here $N_{\text {ex }}=N_{\text {in }}=N$. Hence the total population

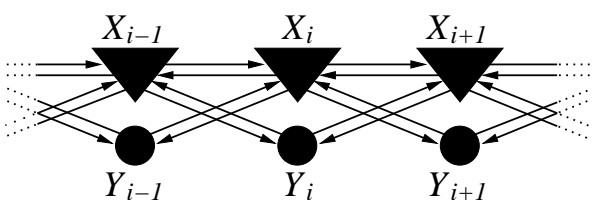

Fig. 3. Dynamical neural network. Excitatory neurons and inhibitory neurons are represented as triangles and circles, respectively. Connections $X_{i} \rightarrow X_{i \pm 1}$ and $X_{i} \rightarrow Y_{i \pm 1}$ are excitatory, whereas connections $Y_{i} \rightarrow X_{i \pm 1}$ are inhibitory.

size is $2 N$. For the numerical simulations described below we take $N=8$. In accordance with Section 2.2, the network has first-neighbor connectivity. The boundary conditions are of the zero-flux type. For this configuration the dynamical equations of the network become

$$
\begin{gathered}
\frac{d X_{i}}{d t}=-\gamma\left(X_{i}-V_{L}\right)-\left(X_{i}-E_{1}\right) \sum_{j=i \pm 1} \omega_{i j}^{(1)} F_{X}\left[X_{j}\left(t-\tau_{i j}\right)\right] \\
-\left(X_{i}-E_{2}\right) \sum_{j=i \pm 1} \omega_{i j}^{(2)} F_{Y}\left[Y_{j}\left(t-\tau_{i j}\right)\right] \\
\frac{d Y_{i}}{d t}=-\gamma\left(Y_{i}-V_{L}\right)-\left(Y_{i}-E_{1}\right) \sum_{j=i \pm 1} \omega_{i j}^{(3)} F_{X}\left[X_{j}\left(t-\tau_{i j}\right)\right] \\
i=1, \ldots, N .
\end{gathered}
$$

Recall also that we consider a spatially homogeneous network, thus $\omega_{i j}^{(I)}=\omega^{(I)}$ and $\tau_{i j}=\tau$. The weights of types (1) and (3) are fixed at $\omega^{(1)}=3.15$ and $\omega^{(3)}=2.5$ respectively. Parameters $\tau$ and $\omega^{(2)}$ can each have different values.

\subsection{Dynamics: routes to spatiotemporal chaos}

The network properties of connection topology and delay in signal transmission turn out crucial for the unfolding of a rich dynamics. It has been noted by several authors that the delay value can serve as a Hopf bifurcation parameter from stationary states into periodic oscillations. Moreover, it provides a route to chaos and a further increase in the chaotic attractor's dimension as its value is incremented. Both mechanisms were identified in our study of neural networks with other configurations and parameters, thus confirming the importance of the delay. Here, we propose a somewhat different, albeit related, route. We fix $\tau=1.8 \mathrm{msec}$ and vary the value of $\omega^{(2)}$, which weighs the amount of inhibitory feedback to the network.

Starting from $\omega^{(2)}=17$ and taking successively lower values of $\omega^{(2)}$, while keeping all other parameters fixed as indicated above, we observe a sequence 
of bifurcations that occur for the neural system. For $16.05<\omega^{(2)}<17$ a stationary state is observed in which the excitatory (resp., the inhibitory) neurons share a common low membrane potential corresponding to a very low firing rate. At $\omega^{(2)}=16.05$, a primary Hopf bifurcation occurs which destabilizes this stationary state giving rise to uniform oscillations of the network with a critical period of $13.76 \mathrm{msec}$. For lower values of $\omega^{(2)}$, the structure of these oscillations becomes increasingly complex. From $\omega^{(2)}=1.69$ downwards, two new dynamical processes take place in conjugation: the network no longer oscillates homogeneously, and a cascade of period-doubling bifurcations is seen for the time-variation of any given neuron's membrane potential. The cascade accumulates for a value of $\omega^{(2)}$ close to 1.641. Past that point, spatiotemporal chaos is observed in a narrow but finite parameter range.

\section{Chaos in the neural model}

By fixing $\omega^{(2)}=1.64$, we can investigate the resulting chaotic regime.

\subsection{Looking at neurons}

The time-variation of each neuron's membrane potential has a bimodal character, with higher frequency oscillations superimposed on the basic cycle (see Fig. 4 - Top). This evolution is aperiodic, and it is one example of rich dy-

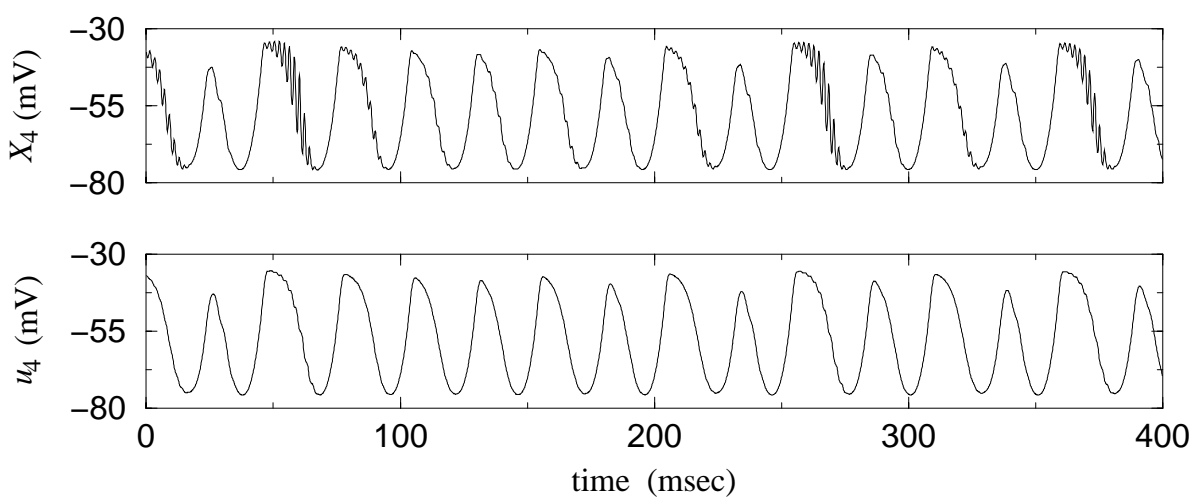

Fig. 4. Top: Chaotic time-series of $X_{4}$, a typical excitatory neuron. Bottom: The corresponding time-series of $u_{4}$. The latter is a low-pass filtered version of $X_{4}$ as explained in the text.

namical behavior not found in networks of simple oscillators.

For the convenience of visualization, we consider "low-pass filters" that eliminate most of the high-frequency behavior. The implementation of the filters 
consists in defining the array of time-averaged variables

$$
u_{i}(t)=\frac{1}{\tau} \int_{t-\tau}^{t} X_{i}(\theta) d \theta
$$

This does not alter the dimension of the dynamics. It just modifies slightly the way we look at the system. We choose $\tau=1.8 \mathrm{msec}$, coinciding with the intrinsic delay in the network as indicated in Section 2.4. This value is approximately $7 \%$ of the average pseudo-period of the cycles in the chaotic regime. Figure 4 - Bottom displays the filtered time-series of a typical excitatory neuron.

\subsection{Looking at spatial modes}

Most of the interesting dynamical behavior that arises through neuron coupling is best evaluated by following entire spatial modes as they evolve in time. Early dynamical exploration could already show that both the network average value of $X$ (or $u$ ) and a profile of local deviations from this average have aperiodic temporal variation. We can make this monitoring more precise and separate the dynamics of the profile of local deviations from that of the spatially averaged network activity. Yet, we remark that the two dynamics are closely coupled in the evolution of the neural network. The deviation profile measures the degree of instantaneous local inhomogeneity, hence spatial structure. The network (spatial) average gives important information about the background activity that the neurons feel, either excitatory or inhibitory. Since we are dealing with spatiotemporal phenomena, we must note that the obtained behavior is conditioned by the moderate size of the network. The spatial correlation length is of the order of the system size. In a larger system, the observed regime would correspond to traveling waves of activity going rightward or leftward in a random fashion, and "riding" on a uniform profile that has itself an irregular temporal variation. In the present network, the waves are not fully developed. Chaos is associated with the destabilization of entire spatial modes. A partial spatial coherence is kept throughout the chaotic regime. This denotes the correlations that exist between neurons, caused by their coupling pattern.

In view of the spatially correlated activity of the neurons, we adopt a spatial mode analysis that had already been useful with other extended systems such as physical systems described by partial differential equations [18]. However, here we are dealing with a spatially discrete system and hence we replace the integrals of [18] with finite sums. As in [18], Fourier spatial modes are chosen for their convenience. The modes are monitored by computing their respective coefficients: 


$$
\begin{aligned}
A_{0}(t) & =\langle u\rangle(t)=\frac{1}{N} \sum_{i=1}^{N} u_{i}(t) \\
A_{j}(t) & =\frac{2}{N-1} \sum_{i=1}^{N} u_{i}(t) \cos \left[j \pi \frac{\left(i-\frac{N+1}{2}\right)}{N-1}\right] \\
B_{j}(t) & =\frac{2}{N-1} \sum_{i=1}^{N} u_{i}(t) \sin \left[j \pi \frac{\left(i-\frac{N+1}{2}\right)}{N-1}\right] \\
j & =1, \ldots, N-1 .
\end{aligned}
$$

Notice that each $u_{i}$ is the instantaneous time-filtered value of an excitatory neuron's membrane potential. The scaling factor $2 /(N-1)$ is arbitrary, since we do not aim at recovering the original function $u_{i}(t) \equiv u(i, t)$ via a sum of the Fourier modes weighted by the $A_{j}$ and $B_{j}$ coefficients. Rather, the $A_{j}$ and $B_{j}$ are taken as the variables spanning a phase-space where the dynamics can be followed. For example, if all $A_{j}$ and $B_{j}$ were constant (i.e., a fixed point in Fourier space), this would correspond to a stationary spatial structure. A limit-cycle in Fourier space, on the other hand, corresponds to a time-periodic spatiotemporal orbit followed by the profile $u_{i}(t), i=1, \ldots, N$.

One may ask what is the minimum number of coefficients required for the dynamics to be adequately monitored. With the present system, a truncation at $j=1$ is enough to characterize the chaotic regime. Figure 5 illustrates the chaotic regime via a projection of the dynamics in Fourier space. The
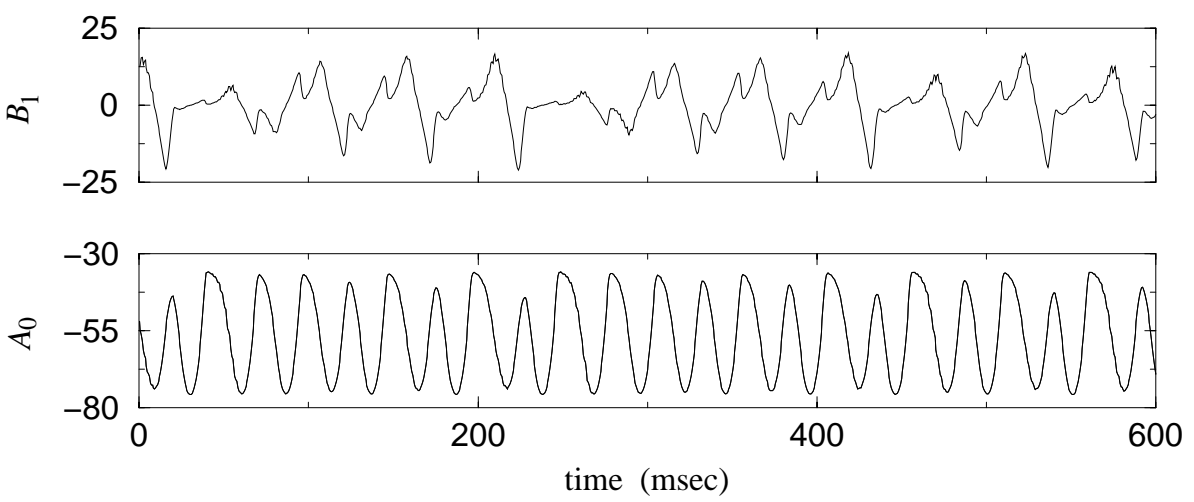

Fig. 5. Chaotic time-series of the Fourier coefficients $A_{0}$ and $B_{1}$, as defined in the main text.

coefficients $A_{0}$ and $B_{1}$ are monitored during an arbitrary period of time. $A_{0}$ is simply the spatially averaged activity of the network. $B_{1}$ is the coefficient of the first sine mode, and already indicates a deviation of the instantaneous activity profile from a homogeneous state. Both coefficients show aperiodic behavior. The coefficients of higher Fourier modes, not shown in the figure, also display irregular variation. 


\subsection{Dynamical reservoir}

Finding an ensemble of UPOs embedded in the chaotic attractor has been one of the objectives of this work. For this system it has been possible to gather evidence that such an ensemble exists, most likely being infinite and countable. However, only the first few UPOs of lowest periods are numerically accessible with moderate effort. If an UPO has a period close to the average pseudo-period of the chaotic attractor, we will say that it is period-one. If the UPO's period is approximately twice the chaotic pseudo-period, then it is period-two, and so on. We could identify with adequate precision, from within the chaotic attractor: 1 period-one UPO, 3 different period-two UPOs, and at least 2 different period-four UPOs. Figure 6 shows one of the period-two UPOs.
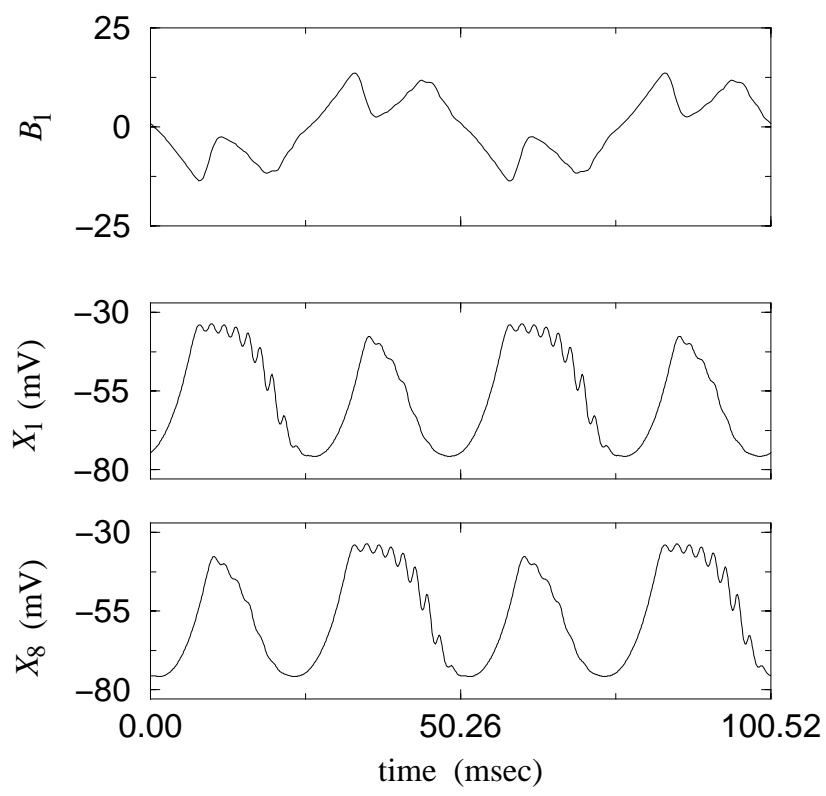

Fig. 6. Period-two unstable periodic orbit embedded in the chaotic attractor. The top of the figure shows the evolution of the Fourier coefficient $B_{1}$ during two complete periods of oscillation. The down-most plots feature the simultaneous variation of the membrane potentials $X_{1}$ and $X_{8}$. The period of the orbit is $T=50.26 \mathrm{msec}$. The spatiotemporal orbit has a reflection symmetry around the central position of the network.

It should be emphasized that UPOs such as the one in Fig. 6 are not spontaneously sustained within the chaotic regime, unless a short timespan is considered during which the system visits the vicinity of the UPO. These UPOs can be found, for instance, using the method outlined in Section 3.4. The latter implies scanning the system's evolution for a very long time, by which it can be detected that an arbitrarily small vicinity of a given UPO is temporarily entered. Instability inevitably leads to the amplification of small differences 
between the system's state and the exact periodic orbit. However, for the purpose of plotting time series such as the ones in Fig. 6, the unperturbed evolution of the system for a limited number of consecutive cycles can provide a sufficiently good approximation for the respective UPO.

Due to the system being spatially extended, the UPOs can display different spatiotemporal symmetries. For example, the orbit in Fig. 6 has the following reflection symmetry: each half of the network has instantaneous activity different from that of the other half, but the neurons are paired such that $X_{1}$ imitates $X_{8}$ from half a period before, $X_{2}$ imitates $X_{7}$ also with a half-period time-lag, and so on. Recall that the total number of excitatory neurons is $N=8$. Other UPOs found within the same attractor do not necessarily obey the left-to-right symmetry as the one of Fig. 6.

\subsection{Tracking the Unstable Periodic Orbits}

The method for tracking UPOs such as the one of Fig. 6, and other UPOs also mentioned in the text, can appear in different variations. In the following we briefly describe a particular procedure which takes advantage of the low dimensionality of the attractor, albeit in the context of an infinite-dimensional system.

Let us consider a Poincaré section at $A_{0}=A_{0}^{P}=-60, \dot{A}_{0}>0$. The time of the $n$th crossing of the Poincaré plane is denoted as $t(n)$. A time-interval variable is defined as $T(n)=t(n)-t(n-1)$. We can look for period-one orbits of the system in the form of fixed points of the map $T(n+1)=\mathcal{F}[T(n)]$, if such a map can be defined. In this case, the period of the orbit and the fixed point have the same numerical value. A period- $k$ orbit with period $T^{*}$ appears as a discrete-time orbit $T_{1}^{*} \rightarrow T_{2}^{*} \rightarrow \ldots T_{k}^{*}$ obeying $\sum_{i=1}^{k} T_{i}^{*}=T^{*}$. However, if the underlying spatiotemporal orbit has the type of reflection symmetry displayed by the UPO of Fig. 6 , it will appear as a period- $k / 2$ orbit of $T(n)$. In the case of Fig. 6, the featured period-two UPO will appear as a period-one orbit of $T(n)$. This can be explained as follows. The homogeneous mode corresponding to $A_{0}$, as well as all the cosine modes corresponding to $A_{j}, j \geq 1$, are invariant with respect to reflection around the central position of the network. The map $\mathcal{F}$ happens to be built upon $A_{0}$, and thus it will treat two halves of a same orbit as being the same if the second half is a spatial reflection of the first. As it comes, it is the durations of each half of such a symmetric orbit that are equal, but via the map $\mathcal{F}$ each of these half-periods of length $T^{*} / 2$ could be interpreted as being the true period of some orbit. Further inspection is required to distinguish between, say, a true period-one UPO and a period-two UPO with reflection symmetry. The first-return map $\mathcal{F}$ is shown in Fig. 7, as well as the second-, third-, and fourth-return maps, respectively, $\mathcal{F}^{(2)}, \mathcal{F}^{(3)}$, 

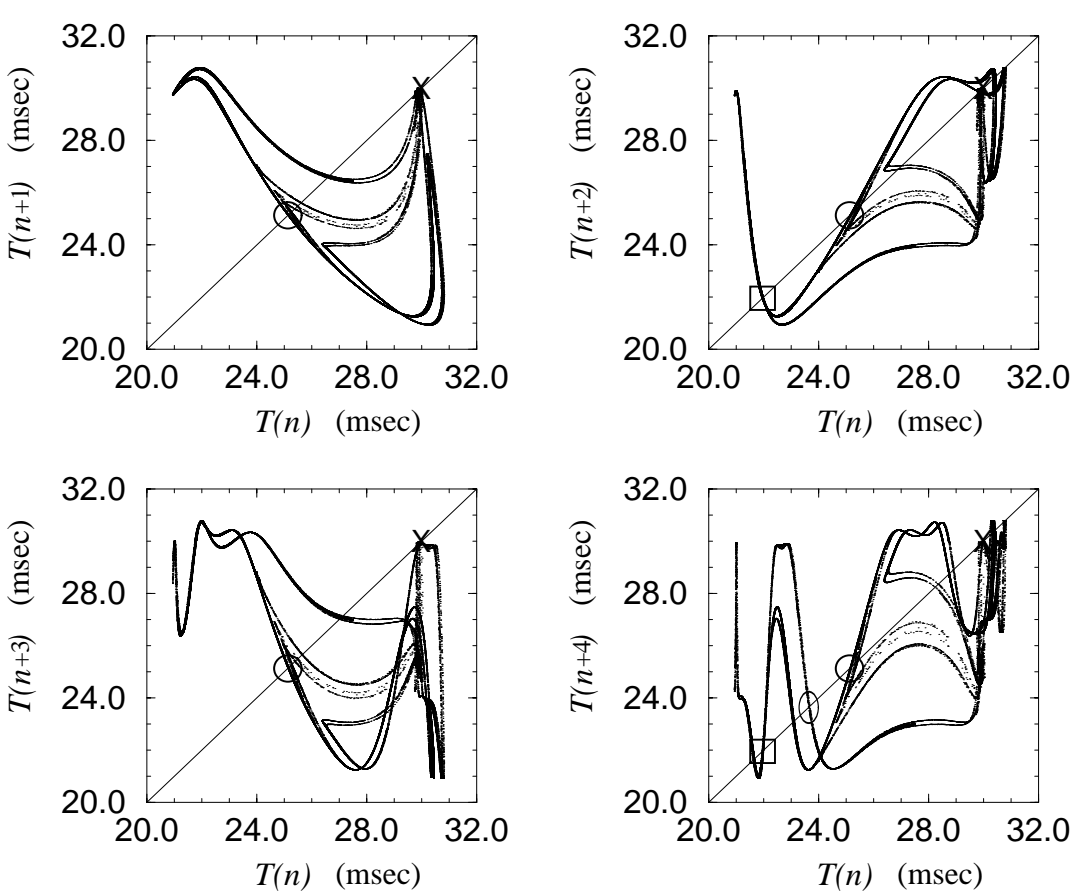

Fig. 7. Return maps of orders $1,2,3$, and 4 , for the time-interval variable $T(n)$ on the Poincaré section $A_{0}=A_{0}^{P}=-60, \dot{A}_{0}>0$. These maps are obtained with the neural network in a chaotic, uncontrolled regime. Shown are the locations of fixed points corresponding to orbits of, respectively, period 1 (X symbol), period 2 (circle and square symbols - these corresponding to different orbits), and period 4 (ellipse symbol). To avoid clutter, no more than one fixed point for any given UPO is shown in each of the four maps. Otherwise, for the period-four orbit, say, we could also plot three other fixed points of the map $\mathcal{F}^{(4)}$, apart from the one signaled by the ellipse. See text for further explanation and Table 1 for numerical values of the fixed points and respective UPOs.

and $\mathcal{F}^{(4)}$. Note that any period-four UPO, say, is a fixed point of the map $T(n+4)=\mathcal{F}^{(4)}[T(n)]$. If, in addition, such UPO displayed spatial reflection symmetry, then it would also be a fixed point of the map $T(n+2)=\mathcal{F}^{(2)}[T(n)]$. It should also be noted that the term "map" is used here in the sense of a multivalued map of a single argument.

It may seem surprising that an infinite-dimensional dynamical system featuring spatiotemporal chaos be described by such low-dimensional maps. Yet, that was already the case with the Kuramoto-Sivashinsky partial differential equation investigated in [18]. In the present paper, the high dimensionality comes also from a spatial distribution, but especially from the presence of delay terms in the evolution equations. Notwithstanding, a few dimensions suffice to describe the attractor, and indeed the maps are low-dimensional projections of the dynamics. However, any perturbation of the system immediately pushes it back into higher dimension. Thus, the apparent simplicity of the maps can be misleading. Nevertheless, a very low-dimensional treatment is possible in this case. Each map features a large number of candidate fixed-points at the 


\begin{tabular}{ccccc}
\hline $\begin{array}{l}\text { Discrete-time } \\
\text { period of the } \\
\text { UPO }\end{array}$ & $\begin{array}{l}\text { Continuous-time } \\
\text { period of the } \\
\text { UPO }(\mathrm{msec})\end{array}$ & $\begin{array}{l}\text { Fixed point } \\
\text { marked in } \\
\text { Fig. } 7(\mathrm{msec})\end{array}$ & $\begin{array}{l}\text { Spatial } \\
\text { reflection } \\
\text { symmetry? }\end{array}$ & $\begin{array}{l}\text { Multiplicity } \\
\text { of the } \\
\text { orbit }\end{array}$ \\
\hline 1 & 29.98 & $29.98(\mathrm{x})$ & yes & 1 \\
2 & 50.26 & $25.13(\mathrm{O})$ & yes & 1 \\
2 & 52.74 & $21.96(\square)$ & no & 2 \\
4 & 104.42 & $23.63(0)$ & no & 2 \\
\hline
\end{tabular}

Table 1

Numerical values associated with the UPOs featured in Fig. 7. The discrete-time period of an UPO is the number of times that the respective trajectory has to recur on the Poincaré plane, after some initial crossing, until all the variables of the dynamics repeat exactly. In general, a period- $k$ orbit gives rise to $k$ fixed points of map $\mathcal{F}^{(k)}$ and also of all maps $\mathcal{F}^{(m k)}$, where $m$ is a positive integer. The exception here is the fixed point $25.13 \mathrm{msec}$ of the second UPO, which is doubly degenerate.

respective period. The majority of them turn out to be false fixed points. A systematic procedure is needed to rule out all of these false candidates: not only do we require that $T(n+k)=T(n)$ for a particular $\mathcal{F}^{(k)}$, but also that a true $k$ th-return is observed in the sense that all monitored variables come back within an arbitrarily small vicinity of their values at $t(n)$. The monitored variables for this purpose can be e.g. the coefficients of the spatial modes up to some adequate order, or the actual values of the neurons' membrane potentials at time $t$ as well as the values at time $t-\tau$. Thus, as mentioned in Section 3.3, six different UPOs could be found. Referring to Fig. 7, Table 1 provides numerical data associated with the six UPOs. The first UPO in the list is a period-one orbit. Inspection of the full dynamics of the network in continuous time reveals that this orbit consists in spatially homogeneous oscillations of the membrane potentials. Trivially, the activity is symmetric with respect to reflection around the central position of the network. The continuous-time period is $29.98 \mathrm{msec}$ which coincides with the numerical value of the respective fixed point of map $\mathcal{F}$. This orbit being period-one, any of its observables will repeatedly assume the same value at successive crossings of the Poincaré plane. In particular, its period of $29.98 \mathrm{msec}$ is itself a fixed point of maps $\mathcal{F}, \mathcal{F}^{(2)}, \mathcal{F}^{(3)}$, and $\mathcal{F}^{(4)}$. It is signaled with a cross $(\mathrm{x})$ in Fig. 7. Recalling previous remarks, such periodic behavior cannot be spontaneously sustained in the chaotic regime, as is the case with the other UPOs also mentioned. The second UPO in the list is a period-two orbit with continuous-time period of 50.26 msec. It is precisely the UPO depicted in Fig. 6. It features a spatial reflection symmetry which was discussed in Section 3.3 and also above in the present section. Hence it appears as a period-one orbit from the viewpoint of map $\mathcal{F}$, implying that it will also give rise to fixed points of maps $\mathcal{F}^{(k)}$ for all $k$. In each case the numerical value of the fixed point thus obtained is 
$50.26 / 2=25.13$ (msec). It is signaled with a circle (o). The third UPO is also period-two, but it does not have the spatial reflection symmetry mentioned above. Its continuous-time period is $52.74 \mathrm{msec}$. This orbit does not give rise to any fixed point of maps $\mathcal{F}$ and $\mathcal{F}^{(3)}$, but it originates two fixed points at map $\mathcal{F}^{(2)}$; since 4 is a multiple of 2 , these two fixed points also appear in map $\mathcal{F}^{(4)}$ as fixed points. Of the two, only the one with numerical value $21.96 \mathrm{msec}$ is explicitly marked, with a square $(\square)$, in maps $\mathcal{F}^{(2)}$ and $\mathcal{F}^{(4)}$ in Fig. 7. It is not hard to see that an UPO which is not symmetric with respect to reflection, such as this one, indeed corresponds to two analogous orbits with exactly the same period and where the activity of each neuron mirrors the activity of the opposite neuron in the related orbit. If we denote the two related UPOs as $O$ and $O_{\text {mirror }}$, respectively, then the activity of, say, excitatory neuron number 1 during one complete period of $O$ will imitate the activity of excitatory neuron number 8 during one complete period of $O_{\text {mirror }}$. The same is true for neurons number 2 and 7, respectively, when compared across the two related UPOs. This imitation is also observed with all other related pairs in our 8-neuron long spatial network arrangement. This is to be expected from symmetry considerations, since a spatially homogeneous network that provides the substrate for some spatially asymmetric UPO, must also display a related UPO which is a spatial reflection of the former UPO. Hence the multiplicity of the representative UPO of period $52.74 \mathrm{msec}$ is taken as 2 . The last orbit in Table 1 is period-four and has a continuous-time period of $104.42 \mathrm{msec}$. Like the previous orbit, it does not have spatial reflection symmetry. Hence it only originates fixed points of map $\mathcal{F}^{(4)}$, and not of map $\mathcal{F}^{(2)}$. Of the four fixed points of this orbit's discrete dynamics on the Poincaré section, only the one with numerical value 23.63 msec is marked over map $\mathcal{F}^{(4)}$ in Fig. 7, with an ellipse (0). For the same reasons that apply to the previous UPO, the representative orbit of period $104.42 \mathrm{msec}$ has multiplicity 2. This raises the total number of UPOs found in this numerical study to six, while not excluding the possibility of finding new UPOs through further exploration.

\section{Discussion}

First we note that the UPO "reservoir" property is indeed found in the chaotic neural attractor. Several different behaviors in the form of UPOs can be identified and described as having different spatiotemporal symmetries. Such a range of behaviors can be useful to perform the kind of dynamical computation referred in Section 1. For instance, neurons at certain parts of the network may provide specialized modulation of input signals, in the spirit of [9]. The orbits that violate the left-to-right spatial symmetry are especially interesting, since they can be used to ensure that the neurons in different parts of the network are consistently doing different things. 
For this procedure to be effective, we need to be able to selectively stabilize the desired UPOs according to the task. If left unperturbed, the chaotic attractor will not spontaneously display such individual orbits in a persistent manner. Hence control methods are required to capture and hold these UPOs, and they should be applied at least for the duration of a computational task. As it comes, stabilization processes that are analogous to those employed with the toy models mentioned in Section 1 can be applied to the present system, which is much more biologically inspired. They share the common feature that the chaotic system is only minimally perturbed, thus no large forcing is required. The control procedure itself is out of the scope of this paper, and will be described in a future publication.

Although we have tried several different network configurations, only an $8+8$ neuron arrangement was chosen to illustrate our ideas in the present paper. The system's spatial extension can be considered as proportional to the number of neurons. In analogy with other spatially extended systems [18], the dimension of the chaotic attractor is expected to grow in monotone fashion with spatial extension. The architecture adopted in the present paper should provide the right balance between small networks, where the dynamics is not complex enough and spatial effects are barely visible, and very large networks, where chaos can be too much developed and numerically difficult to assess in a first approach in view of computation.

It is demonstrated that a network of model neurons akin to leaky integrators, with physically plausible nonlinear connectivity and intrinsic delays in signal transmission, is capable of displaying a rich set of behaviors without the need of any significant parameter deviation from reference values. The model, which is amenable to electronic implementation, takes advantage of a fully time-continuous dynamics together with spatial distribution, thus providing an adequate framework for the dynamical computation paradigm that motivated this work. A reservoir of behaviors exists in chaos, which could be explored for the sake of computation. Here the reservoir is spatiotemporal, and the worthiest candidates for practical applications would be the ones taking advantage of this.

\section{Acknowledgment}

The author wishes to thank the anonymous reviewers for their useful comments and suggestions. 


\section{References}

[1] A. Babloyantz, J. Salazar and C. Nicolis, Evidence of chaotic dynamics of brain activity during the sleep cycle, Physics Letters A, 111: 152-156, 1985.

[2] P. Rapp, I. Zimmerman, A. Albano, G. de Guzman and N. Greenbaun, Dynamics of spontaneous neural activity in the simian motor cortex: The dimension of chaotic neurons, Physics Letters A, 110: 335-338, 1985.

[3] M. Conrad, What is the use of chaos? In A. Holden, editor, Chaos, Manchester University Press, Manchester UK, pages 3-14, 1986.

[4] W. Freeman, Simulation of chaotic EEG patterns with a dynamic model of the olfactory system, Biological Cybernetics, 56: 139-150, 1987.

[5] W. Freeman, The physiology of perception, Scientific American, 264: 78-85, 1991.

[6] A. Babloyantz and C. Lourenço, Computation with chaos: A paradigm for cortical activity, Proceedings of the National Academy of Sciences USA, 91: 9027-9031, 1994.

[7] A. Babloyantz and C. Lourenço, Brain chaos and computation, International Journal of Neural Systems, 7:461-471, 1996.

[8] N. Crook and T. Scheper, A Novel Chaotic Neural Network Architecture. In M. Verleysen, editor, proceedings of the $9^{\text {th }}$ European Symposium on Artificial Neural Networks (ESANN 2001), D-Facto pub., pages 295-300, April 25-27, Bruges (Belgium), 2001.

[9] C. Lourenço, Attention-locked computation with chaotic neural nets, International Journal of Bifurcation and Chaos, 14:737-760, 2004.

[10] N. Crook, W. J. Goh and M. Hawarat, The nonlinear dynamic state neuron. In M. Verleysen, editor, proceedings of the $13^{\text {th }}$ European Symposium on Artificial Neural Networks (ESANN 2005), d-side pub., pages 37-42, April 27-29, Bruges (Belgium), 2005.

[11] H. Jaeger, The "echo state" approach to analyzing and training recurrent neural networks, GMD Report 148, German National Research Center for Information Technology, 2001.

[12] W. Maass, T. Natschläger and H. Markram, Real-time computing without stable states: A new framework for neural computation based on perturbations, Neural Computation, 14:2531-2560, 2002.

[13] L. Kaczmarek, A model of cell firing patterns during epileptic seizures, Biological Cybernetics, 22:229-234, 1976.

[14] A. Destexhe, Ph.D. Dissertation, Université Libre de Bruxelles, March 1992.

[15] D. Kleinfeld, Sequential state generation by model neural networks, Proceedings of The National Academy of Sciences USA, 83:9469-9473, 1986. 
[16] I. Tokuda, R. Tokunaga and K. Aihara, Back-propagation learning of infinitedimensional dynamical systems, Neural Networks, 16:1179-1193, 2003.

[17] C. Lourenço, Dynamical reservoir properties as network effects. In M. Verleysen, editor, proceedings of the $14^{\text {th }}$ European Symposium on Artificial Neural Networks (ESANN 2006), d-side pub., pages 503-508, April 26-28, Bruges (Belgium), 2006.

[18] C. Lourenço, M. Hougardy and A. Babloyantz, Control of low-dimensional spatiotemporal chaos in Fourier space, Physical Review E, 52:1528-1532, 1995. 